\title{
Horn effect of tire/road noise: Modelling and experiments of acoustic network resonators in horn-like structures
}

\section{Bin Wang}

Ecole des Ponts ParisTech, Laboratoire Navier, ENPC-IFSTTAR-CNRS-UPE, UMR 8205, 6-8 avenue Blaise Pascal, 77455 Marne la Vallée, France

E-mail: bin.wang@enpc.fr

\section{Denis Duhamel}

Ecole des Ponts ParisTech, Laboratoire Navier, ENPC-IFSTTAR-CNRS-UPE, UMR 8205, 6-8 avenue Blaise Pascal, 77455 Marne la Vallée, France

E-mail: denis.duhamel@enpc.fr

\begin{abstract}
The noise generated in the contact zone can be amplified by the horns made by the surfaces of the tire and the road. Previous researches on the horn effect have only investigated smooth tires and roads. In this work we take into account the tire treads in the calculation of the horn effect. The air resonances and horn effects are studied in the same model. A model of flanged networks is established for the tire/road system, and multi-domain coupling methods are developed for the calculation of the acoustic fields around the flanged networks. With this model the reductions of the amplifications of the horn effect by the networks can be estimated. The model of the flanged networks are also proved to be effective by the experiments. Wooden pipes between a wooden cylinder and a sheet of plywood are built for the validation. A real tire on a sheet of plywood is also measured and calculated with the proposed methods.
\end{abstract}

Keywords: Tire; Acoustics; Noise; Horn; Amplification; Diffraction; Radiation; Modelling; Network; Pipe; BEM; Measurement; Experimental result.

\section{Introduction}

Road traffic noise is a part of the community noise which also includes other traffic, industries, construction, public work and so on see Berglund et al (1999). Among these noise sources, the road traffic noise is a dominant source. About $40 \%$ of the Europeans are exposed to levels of road traffic noise exceeding $55 \mathrm{dBA}$ daytime, and $20 \%$ are exposed to levels exceeding 65dBA according to the study by Lambert and Vallet (1994). Nowadays, this problem is even worse due to the population growth, urbanization and the enlargements 
2

author

of the highway systems. The traffic noise is very annoying and has many adverse health effects. It can cause population annoyance, interference with communication and intended activities, disturbance of sleep, hearing impairment and so on. It also has large economic effects. From the EU Green Paper by the European commission (1996) we know that the cost of traffic noise in 17 European countries is about $0.65 \%$ of GDP.

The traffic noise emitted to the environment includes the tire/road noise, powertrain noise and the aerodynamic noise, see Qatu et al (2009); Qatu (2012). The contribution of the tire/road noise in the total noise level is the largest for cars travelling faster than $50 \mathrm{~km} / \mathrm{h}$ and trucks travelling faster than $80 \mathrm{~km} / \mathrm{h}$ see Bernhard et al (2005); Gagen (1999). The tire/road system can be seen as a horn-like structure. The surfaces of the tire and the road constitute horns in front of and behind the contact zone. The noise generated in the contact zone is amplified by the horn-like structures. This horn effect was studied by several authors. Two-dimensional computations of the horn effect were done by Anfosso-Ledee et al (2000b) and compared to three-dimensional results in Anfosso-Ledee et al (2000a). A detailed analytical, numerical and experimental analysis of the horn effect was done by Graf et al (2002); Kuo et al (2002). The analytical model of a sphere on an absorbing ground was used by Bravo and de la Colina (2015); Bravo (2017) and compared to measurements to estimate the horn effect on a finite impedance ground. While other studies were in the frequency domain, Zhang et al (2017) used efficient computations in the time domain to predict the horn effect. All these studies assume a smooth surface for the tire and the road.

The acoustic fields around the tire/road system can be influenced by the network resonances in the contact zone. These come from the sculpture of the tread and the irregular level of the road which creates cavities in the contact zone. It is known that this generates air-pumping but this can also have influence on the horn effect itself since this horn effect is rather sensible to the true geometry of the horn. This effect will be studied in the present work. Horn-like structures with networks inside can be considered as bodies with macro porosities, which can be seen in the analysis of porous walls, horn effects of road/tire and so on. Such problems include the acoustic transmission-radiation problems. The porosities could be made of networks, several parallel pipes or a mixture of them. In such systems the networks to be analysed have small cross sections. However, the cross sections are neither as small as the arbitrary microscopic holes in many porous materials nor potentially as complicated as some pipes in mufflers. Mufflers can have complicated internal structures such as inlet/outlet tubes, thin baffles, perforated tubes, and sound absorbing materials. So it is not suitable to use the same methods to calculate the sound pressure fields. Besides, in porous materials acoustic energy is dissipated as heat because of viscosity, so noise could be reduced by porous materials. But for the networks considered in this work the main mechanisms of noise reduction by networks are the pipe resonances. However, the methods for mufflers could give some inspirations. There are several methods for the analysis of mufflers summarized in Park et al (2009). The multi-domain boundary element method (BEM) and BEM with the transfer matrix could be used for the calculation of the flanged network, but some changes should be made.

A brief introduction of the applications of multi-domain BEM can be seen in $\mathrm{Wu}$ (2008). It was first used to analyze the potential problem and elasticity by Brebbia and Walker (1980). Then it was introduced to solve acoustic problems. An important application of multi-domain BEM to acoustics is the coupled interior/exterior problems of Cheng (1988) where the interior boundary integral equation is used for a finite internal domain and the exterior boundary integral equation is used for an infinite external domain. The solution at the interface between the two domains is coupled by the continuity constraint equations. 
So the problem can be solved as a continuous field problem to get a solution at any point in the whole domain. The multi-domain BEM is also used to deal with problems with several acoustic media in Utsuno et al (1990). Another common application is the problem about thin bodies considered by Cheng et al (1991); Wang et al (1993) before using the hypersingular integral equation.

Concerning the interior network, transfer matrix techniques have been applied to the analysis of connected pipes with many changes of sectional area in Munjal (1987). The advantage is that only two-by-two matrices are needed and it is possible to work on a desktop computer, but only using the transfer matrix is not easy to apply to networks with complicated junctions. In Craggs and Stredulinsky (1990) a different form of transfer matrix is used, together with matrices derived for two-dimensional junctions by the finite element method, to describe a complete network. In Lou et al (2003) the transfer matrix is used to combine the impedance matrix of each substructure of a silencer. The transfer matrix can easily connect two substructures and describes the relation in the pressures and particle velocities between them. But an assumption should be true to get the transfer matrix, which is the plane wave propagation in the connected parts.

For the network analysed in this paper, BEM of one computational domain is available, but only for very simple cases, as BEM needs fine mesh around the resonant frequency of the air in the network to get converged solutions. Substructuring techniques can reduce the matrix size and the total computational time for complex structures. Dividing the whole acoustic domain into several subdomains is the main idea of the multi-domain coupling method. Each subdomain, which could be an exterior or an interior one, has a well-defined boundary and the same material property. The exterior boundary integral equation is used for the infinite external subdomain. For the interior subdomain, as the networks studied here will remain simple, transfer matrices with $2 \mathrm{D}$ analytical relations will be used. The solutions at the interfaces between the subdomains are coupled by the continuity constraints of the sound pressure and normal particle velocity.

First the model of flanged networks and the computational methods are introduced. Then simulations and experiments are compared to check the accuracy of the proposed methods in the following section. Next the model and the computational methods are applied to a real tire. Last some conclusions are drawn.

\section{Modelling of network resonators in horn-like structures}

Our main objective is to understand the influence of cavities in the contact zone on the sound radiation and the horn effect for tire/road noise. The structures that will be studied looks like that of Fig.1a and consists in a body diffracting a sound pressure created by sources in air. In contrary to the classical case where for instance a velocity boundary condition is applied on the whole surface of the body, here the body has an internal structure made by a network of waveguides such that different points at the surface of the body can be connected by this network. The network constituting the porosities could have any connection pattern: an arbitrary network, parallel pipes or a mixture of them. The pipes here have small cross sections. The purpose of the present work is to estimate the influence of such a network on the diffraction of sound by the body compared to the case where there is no network and to apply this to the case of a tire on a road. 


\subsection{Problem specification}

Fig.1a represents a three dimensional network inside a body with arbitrary flanges. A point source $S$ is put near the flange. The acoustic wave at the receiver point $R$ includes three parts (see Fig.1a): a wave directly from the source, a wave reflected from the flanged without network (see Fig.1b) and a wave radiating from the network with flange (see Fig.1c).

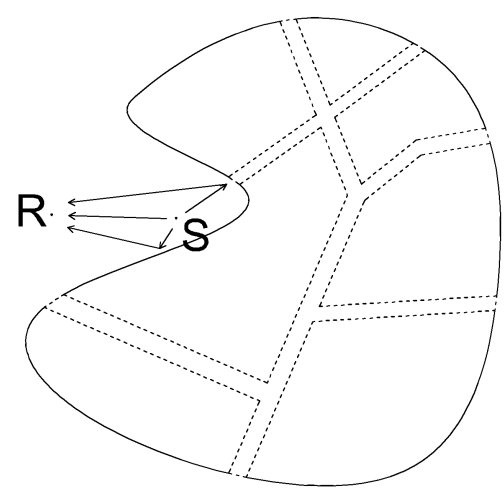

(a)

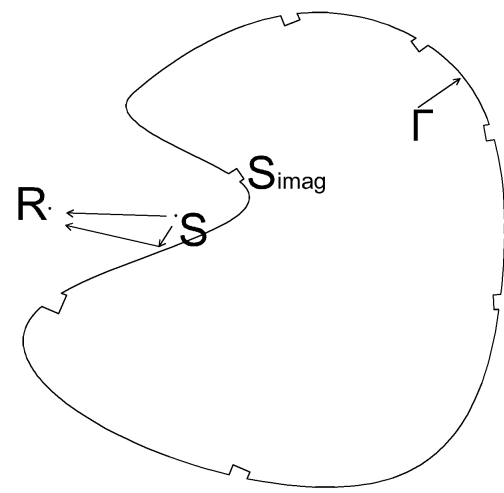

(b)

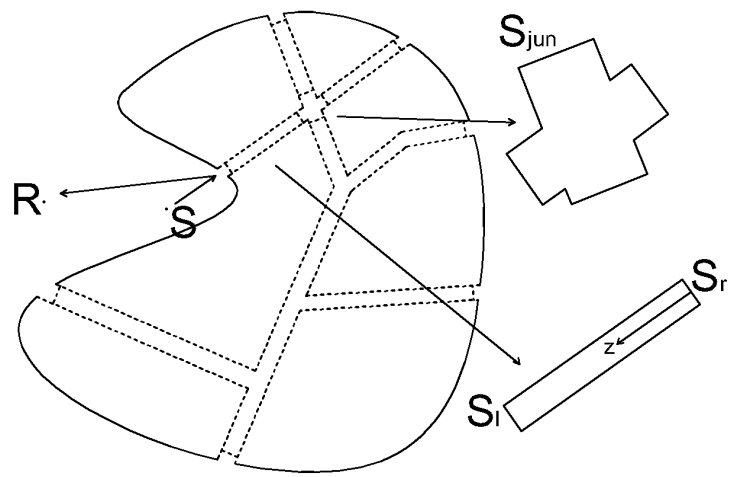

(c)

Figure 1: (a) Total pressure; (b) Pressure directly from the source and reflected by the flange; (c) Pressure radiating from the network

The wave from the source arrives at ends of pipes and then propagates in the network. The straight parts of the network are very thin compared with the wavelength to be analyzed. The frequencies of the source are below the cut off frequencies of straight parts. So there is only a plane wave motion in the straight parts that consists of a forward travelling wave and a backward reflected wave. The waves in the junctions of the network have higher order modes generated by the geometrical discontinuities, but they don't propagate in the straight parts where they are quickly attenuated outside the immediate proximity of the junctions.

In Fig. 1b, imaginary pipe ends are used instead of the real ends. The imaginary ends are inside the network but close to the real ones. The imaginary surfaces $S_{i m a g}$ are assumed to be perpendicular to pipes walls to simplify but any surface not to close to the opening could be used. Thus, at $S_{i m a g}$ the wave is uniform and the velocity is perpendicular to 
the imaginary end, which is useful in the method proposed in this paper. However, it is not uniform at the real ends, even if the real ends are perpendicular to the network walls, because the cross sections change suddenly. This is why imaginary surfaces $S_{i m a g}$ are used.

Concerning the boundary conditions, we assume here that the surface of the body is rigid, except at the pipe ends $S_{\text {imag }}$ where the pressure and the velocity are continuous between the pipe ends and the body surface. All other surfaces such that the network walls and the surface of the road, if there is one, are also rigid. It would not be too difficult to consider impedance boundary conditions but to simplify this is not done in this work.

\subsection{Multi-domain coupling methods}

The network to be analyzed consists of cylindrical or rectangular pipes with small cross sections. The calculation of the total acoustic pressure $p_{t o t}$ at a point $R$ in the exterior domain in Fig.1a by multi-domain coupling methods is introduced in this section.

In boundary element methods, see Brebbia and Walker (1980); Ciskowski and Brebbia (1991); Chen and Zhou (1992); Bonnet (1999), for a problem with an unbounded domain, the integral equation to be solved is given by

$$
c_{e}(\mathbf{x}) p(\mathbf{x})=\int_{\Gamma} p(\mathbf{y}) \frac{\partial G}{\partial \mathbf{n}_{y}}(\mathbf{x}, \mathbf{y}) d \mathbf{y}-\int_{\Gamma} \frac{\partial p}{\partial \mathbf{n}_{y}}(\mathbf{y}) G(\mathbf{x}, \mathbf{y}) d \mathbf{y}+p_{i n c}(\mathbf{x})
$$

$\Gamma$ includes the flange and the imaginary network ends (see Fig.1b). $y$ is a point on the boundary $\Gamma$ and $\mathbf{x}$ can be a point on the boundary or in the fluid domain. $p_{i n c}(\mathbf{x})$ is the incident pressure from the source without the structure. $G$ is the Green function such that in free field $G=\frac{e^{i k r}}{4 \pi r}$ with $k$ the wavenumber and $r=|\mathbf{x}-\mathbf{y}|$ the distance between the point source and the receiver. In case of a rigid road, the contribution of the image source relative to the road should be included in the Green function to avoid the mesh of the ground. $\mathbf{n}$ is the unit normal vector pointing into the fluid domain. To get the total pressure $p_{t o t}$ in the exterior domain, let $c_{e}(x)=1$, and the pressure $p(\mathbf{y})$ and its derivative $\frac{\partial p}{\partial \mathbf{n}_{y}}$ on the surface $\Gamma$ should be calculated first.

To get the pressure $p(\mathbf{y})$ and its derivative $\frac{\partial p}{\partial \mathbf{n}_{y}}$ on the surface $\Gamma$ of a complex network with flange, the computational domain in Fig.1 a should be divided into an exterior subdomain and an interior subdomain by creating imaginary ends for the network. The exterior subdomain is solved by BEM to get BEM system matrices and an excitation vector. The interior subdomain is solved by analytical methods (transfer matrix) to get the relation between $p$ and $q=\frac{\partial p}{\partial \mathbf{n}}$ at these ends. Then the exterior and interior subdomains are coupled at the interfaces. Finally, by solving the overall equation system, one has $p(\mathbf{y})$ and $\frac{\partial p}{\partial \mathbf{n}_{y}}$ on the surface $\Gamma$. The process is described in detail in the following.

\subsubsection{Exterior subdomain}

In Eq. (1), for a point $\mathbf{x}$ on $\Gamma, c_{e}(\mathbf{x})$ equals $1 / 2$ if the surface $\Gamma$ is regular at this point. The discretization of Eq. (1) is obtained from a mesh of the surface of the domain. Then a linear system (2) can be obtained whose solution gives an approximation of the solution on the surface $\Gamma$. More information can be found in Duhamel $(1995,1994)$.

$$
\mathbf{A P}+\mathbf{B Q}=\mathbf{P}_{\text {inc }}
$$


$\mathbf{P}, \mathbf{Q}$ and $\mathbf{P}_{i n c}$ are vectors of pressure, derivative of pressure and incident pressure, respectively. $\mathbf{A}$ and $\mathbf{B}$ are BEM system matrices.

For the exterior subdomain in Fig.1b, dividing the vectors in Eq. (2) into vectors of imaginary ends and vectors of flange, one has

$$
\mathbf{A}_{E}\left[\begin{array}{c}
\mathbf{P}_{e p}^{E} \\
\mathbf{P}_{f}^{E}
\end{array}\right]+\mathbf{B}_{E}\left[\begin{array}{c}
\mathbf{Q}_{e p}^{E} \\
\mathbf{Q}_{f}^{E}
\end{array}\right]=\left[\begin{array}{c}
\mathbf{P}_{i n c}^{e p} \\
\mathbf{P}_{i n c}^{f}
\end{array}\right]
$$

The subscripts and superscripts $e p$ and $f$ mean the imaginary ends of the pipes and flange, respectively, and $E$ means exterior. Matrices $\mathbf{A}_{E}$ and $\mathbf{B}_{E}$ can be obtained by solving the problem in Fig.1b with BEM software. In the BEM software, using the rigid boundary condition on the surface $\Gamma$, one can get $\mathbf{A}_{E}$. Using the soft boundary condition, one can get $\mathbf{B}_{E}$. The incident pressure $p_{\text {inc }}$ can be obtained in either of the two computations above.

\subsubsection{Interior subdomain}

\section{One dimension without loss}

The straight pipe through the flange or the straight part of a network between the flange and a junction or two junctions in Fig.1c, whose central axis is labelled as $z$, is thin compared to the wavelength to be analyzed. There is only plane wave consisting of a forward travelling wave and a backward reflected wave. Pressure $p$ and its derivated $q$ are constant on a plane perpendicular to $z$. So one has the wave equation (4) and its solution (5) and (6).

$$
\frac{\partial^{2} p}{\partial z^{2}}+k^{2} p=0
$$

$k=\frac{\omega}{c}$ is the wave number, $\omega$ the angular frequency and $c$ the speed of sound.

Suppose that the solution of equation (4) is

$$
p(z)=a \cos k z+b \sin k z
$$

The convention $e^{-i \omega t}$ is adopted, where $i^{2}=-1$ and $\rho$ is the density of air. The velocity is given as

$$
v(z)=\frac{1}{i \rho \omega} \frac{\partial p}{\partial z}
$$

Surfaces $S_{r}$ and $S_{l}$ in Fig.1c have different normal directions. For one node on $S_{r}$ $\left(z=z_{r}\right), p$ and $q$ can be written as, see Fig.2

$$
\begin{aligned}
& p_{r}=a \cos k z_{r}+b \sin k z_{r} \\
& q_{r}=k a \sin k z_{r}-k b \cos k z_{r}
\end{aligned}
$$

On $S_{l}\left(z=z_{l}\right)$

$$
\begin{aligned}
& p_{l}=a \cos k z_{l}+b \sin k z_{l} \\
& q_{l}=-k a \sin k z_{l}+k b \cos k z_{l}
\end{aligned}
$$




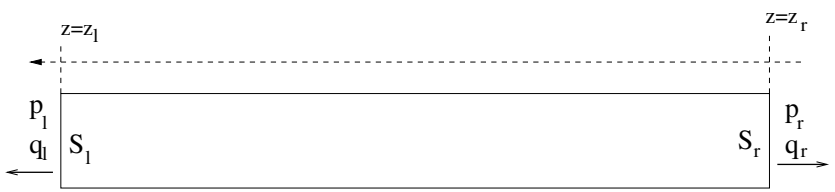

Figure 2: Straight pipe with the pressure and its derivative at the extremities

One gets $a$ and $b$ from equation (7), and then substitute them into (8). From this relation one has to define a link between the values of pressure and its normal derivative for the nodes at both ends of the pipe to complete relation (3). The relation of $\mathrm{p}$ and q between one node on $S_{r}$ and another node on $S_{l}$ can be obtained as

$$
\left[\begin{array}{l}
q_{r} \\
q_{l}
\end{array}\right]=\left[\begin{array}{l}
t_{11} t_{12} \\
t_{21} t_{22}
\end{array}\right]\left[\begin{array}{l}
p_{r} \\
p_{l}
\end{array}\right]
$$

Taking the pressure as the primary variable and expressing the normal derivative in term of the pressure, in (9), $p$ can be expressed as the mean value of the pressure at nodes in a section because the pressure should be constant at each pipe end. So $q$ of any node $i$ on $S_{r}$ and $q$ of any node $j$ on $S_{l}$ becomes

$$
\left[\begin{array}{l}
q_{r i} \\
q_{l j}
\end{array}\right]=\left[\begin{array}{ll}
t_{11} & t_{12} \\
t_{21} & t_{22}
\end{array}\right]\left[\begin{array}{c}
\frac{1}{n_{r}} \sum_{i=1}^{i=n_{r}} p_{r i} \\
\frac{1}{n_{l}} \sum_{j=1}^{j=n_{l}} p_{l j}
\end{array}\right]
$$

Here $n_{r}$ and $n_{l}$ are the node numbers at each end. Thus, the relation between the $p$ vector $\mathbf{P}_{e}$ and $q$ vector $\mathbf{Q}_{e}$ at the two ends can be written as

$$
\mathbf{Q}_{e}=\mathbf{S P}_{e}
$$

where the transfer matrix

$$
\mathbf{S}=\left[\begin{array}{ll}
\frac{t_{11}}{n_{r}} \mathbf{J}_{n_{r}, n_{r}} & \frac{t_{12}}{n_{l}} \mathbf{J}_{n_{r}, n_{l}} \\
\frac{t_{21}}{n_{r}} \mathbf{J}_{n_{l}, n_{r}} & \frac{t_{22}}{n_{l}} \mathbf{J}_{n_{l}, n_{l}}
\end{array}\right]
$$

$\mathbf{J}_{i, j}$ is a matrix with $i$ lines and $j$ columns, where each element equals one.

For a body with parallel pipes, one has the system equations (3) for the exterior subdomain and the analytical relation (11) for the two ends of each straight pipe. Therefore the overall system can be obtained. After applying the boundary conditions to the flange and tube walls and solving the overall system, the values of $p$ and $q$ for each node on the whole surface $\Gamma$ can be obtained. 


\section{One dimension with losses}

According to the study by Kinsler et al (1999), there are viscous losses at rigid walls of thin pipes. In this case, the equation of motion is

$$
-\frac{\partial p}{\partial z}=-i \rho \omega \bar{v}+R \bar{v}
$$

with the average velocity $\bar{v}$ at the pipe cross-section, and the damping coefficient of the thin pipe

$$
R=\frac{1}{r} \sqrt{2 \eta \rho \omega}
$$

where $r$ is the radius of the circular pipe, and $\eta$ is the coefficient of shear viscosity.

The equation of continuity and equation of state do not change, then the wave equation (15) can be obtained

$$
\frac{\partial^{2} p}{\partial z^{2}}+k^{2} p+\frac{i \omega R p}{\rho c^{2}}=0
$$

The solution of equation (15) can be given as

$$
p(z)=a \cos k^{\prime} z+b \sin k^{\prime} z
$$

with $k^{\prime}=k+i \alpha$, where $\alpha$ is the absorption coefficient for the viscous losses

$$
\alpha=\frac{1}{r c} \sqrt{\frac{\eta \omega}{2 \rho}}
$$

The average velocity can be obtained from equation (13)

$$
\bar{v}=\frac{1}{i \rho \omega-R} \frac{\partial p}{\partial z}
$$

Equations, which are similar to equations (7)-(12), can be obtained, in which $k$ should be replaced by $k^{\prime}$. So an equation similar to equation (11), describing the relation between the $p$ vector $\mathbf{P}_{e}$ and $q$ vector $\mathbf{Q}_{e}$ at the two ends, can be written as

$$
\mathbf{Q}_{e}=\mathbf{S}^{\prime} \mathbf{P}_{e}
$$

where in matrix $\mathbf{S}^{\prime}$, wave number $k$ is replaced by $k^{\prime}$.

The impedance of the pipe with viscous losses is

$$
Z_{v}=\frac{l}{\pi r^{3}} \sqrt{2 \eta \omega \rho}-\frac{\rho l}{\pi r^{2}} \omega i
$$

where $l$ is the pipe length. 
Thermal conduction losses should also be taken into account in thin pipes besides viscous losses according to Kinsler's measurements. The coefficient of shear viscosity $\eta$ used above should be replaced by an effective coefficient $\eta_{e}$. The latter is defined by

$$
\eta_{e}=\eta\left[1+\frac{\gamma-1}{\sqrt{P_{r}}}\right]^{2}
$$

where $\gamma$ is the ratio of specific heats of air and $P_{r}$ is the Prandtl number.

The overall system can be obtained from equations (3) for the exterior subdomain, from (19) for the pipes which includes the viscous and thermal conduction losses and then solved as previously.

\section{Two dimensions}

In this part, all the pipes and junctions constituting a network are in the same plane and have the same width. Only rectangular pipes and three types of junctions will be considered, which are $\mathrm{L}$ shaped, $\mathrm{T}$ shaped and cross junctions. The straight pipes have sufficiently small transverse dimensions compared to the wave length under consideration, so the higher order waves can not propagate. However, the higher order modes are required in order to satisfy the boundary conditions imposed by the junctions.

Miles first introduced the transmission line and impedance analogies for plane discontinuities in the articles Miles (1946a) and Miles (1946b). Then Miles (1947) gave the relations between pressures and velocities of right-angled joint ends in rectangular pipes, which can be used to get the boundary conditions at the junction ends. In Miles theory the propagation of the principal wave is represented by the voltage and current on a transmission line. An impedance element of the equivalent circuit for the junctions is calculated to give the effect of the high order modes on the principal wave.

The junction under consideration is shown in Fig.3. There are $n$ straight pipes and one has $n=2$ for the $\mathrm{L}$ junction, $n=3$ for the $\mathrm{T}$ junction and $n=4$ for the cross junction. The problem is considered as 2D, for the incident waves are assumed to be plane and the depth of the pipes is irrelevant. The voltages and currents are respectively denoted by $U_{0}$ and $I_{0}$ at the junction ends. They are assumed related through the admittance matrix $\left(Y_{i j}\right)$ such that

$$
I_{0}^{i}=\sum_{j=1}^{n} Y_{i j} U_{0}^{j} \quad i=1,2, \ldots, n \quad(n=2,3 \text { or } 4)
$$

The equivalent circuits of the junctions and the calculation of $Y_{i j}$ can be seen in Miles (1947) where one can see the terms of high order modes of $Y_{i j}$. Here only the first approximations of $Y_{i j}$ are given.

$$
\begin{aligned}
& Y_{12}=Y_{21}=Y_{23}=Y_{32}=Y_{34}=Y_{43}=Y_{14}=Y_{41}=i Y_{0} \theta^{-1} \\
& Y_{13}=Y_{31}=Y_{24}=Y_{42}=i Y_{0} \csc \theta \\
& Y_{11}=Y_{22}=Y_{33}=Y_{44}=i Y_{0} \cot \theta
\end{aligned}
$$

With $Y_{0}=\rho c / S$ and $\theta=k a . S=a^{2}$ is the straight pipe cross section area.

At the junction ends, one has the relations

$$
p=\sqrt{S} I_{0}
$$




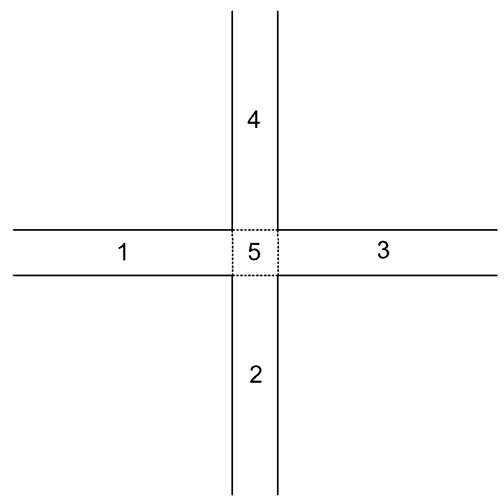

Figure 3: Junction

$$
v=\sqrt{S}^{-1} U_{0}
$$

Thus, one has the relations between pressures and velocities at the junction ends.

For a body with a 2D network, one has equations (22), (24) and (25) for each junction, the analytical relation (11) or (19) for the two ends of each straight pipe and the system equations (3) for the exterior subdomain. All this can then be solved as previously.

\section{Simulation and experimental validation}

\subsection{Simulations}

In this section, we calculate the acoustic fields of pipes between a round surface and a plane surface as in Fig.4a. The round surface in this section is a smooth cylinder. Its radius is $0.27 \mathrm{~m}$ and its width is $0.15 \mathrm{~m}$. The contact zone between the cylinder and the plane surface is $0.15 \mathrm{~m} \times 0.1 \mathrm{~m}$.

\subsubsection{Straight pipe}

In the contact zone in Fig.4a, we first study the case where there is a longitudinal straight pipe at the center. The pipe cross-section is $0.005 \mathrm{~m} \times 0.01 \mathrm{~m}$. Since the pipe is very simple, for this case, we can use BEM directly to get the acoustic fields by meshing the cylinder and the straight pipe first. The meshes can be seen in Fig.5a. Since the cylinder has a symmetric plane, half of the cylinder is meshed.

The source is at $(0.1 \mathrm{~m}, 0 \mathrm{~m}, 0.005 \mathrm{~m})$. From the reference system of Fig. $4 \mathrm{~b}$, the source is thus at $0.1 \mathrm{~m}$ on the right of the center of the contact zone and at $5 \mathrm{~mm}$ above the ground. The results at $(1 \mathrm{~m}, 0 \mathrm{~m}, 0.06 \mathrm{~m})$ can be seen in Fig.6. The solid line, dash line and dot line are results for no pipe, one straight pipe without losses and one straight pipe with viscous losses respectively. The viscous losses are taken into account in the BEM calculations by an impedance which is given by equation (20). The difference of SPL between the solid line and the dot line is $5.5 \mathrm{~dB}$ at the resonant frequency $1430 \mathrm{~Hz}$, and is $6.8 \mathrm{~dB}$ between the solid line and dash line at the resonant frequency $1460 \mathrm{~Hz}$. The resonant frequency has a small shift of $30 \mathrm{~Hz}$. By using the viscous losses, the differences become smaller. 


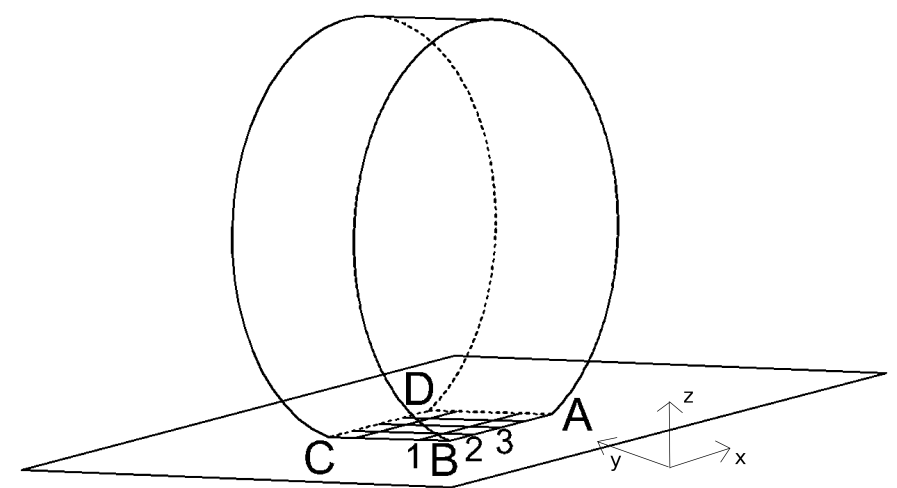

(a)

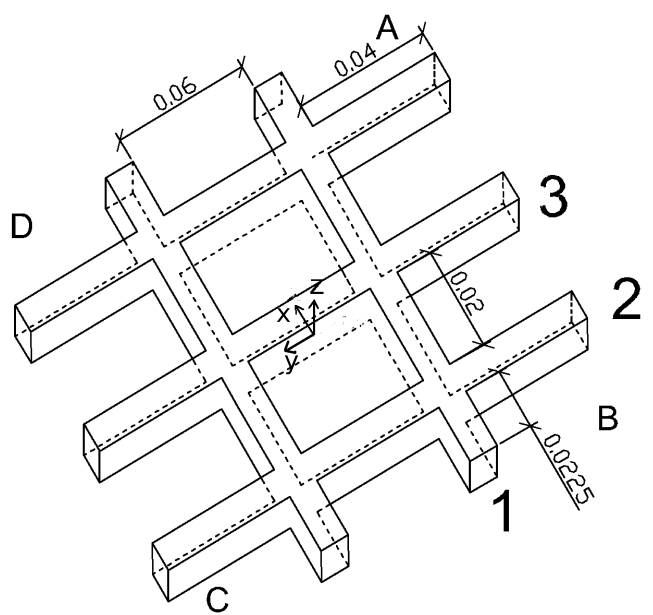

(b)

Figure 4: (a) Network between a round surface and a rigid plane surface; (b) The network with open ends.

The numerical results of this example will be compared with measurements in section 3.2.3.

\subsubsection{Network}

In the contact zone in Fig.4a, there is a network at the center which can be seen in Fig.4b. In the network, there are two longitudinal (x direction in Fig.4b) and three transverse branches (y direction). The network has symmetric planes $x z$ and $y z$. The plane surface is in the plane $x y$. The pipe width is $0.009 \mathrm{~m}$. Since the cylinder has a symmetric plane $x z$, half of the cylinder without network is meshed with $2 \mathrm{D}$ elements. The source is at $(0.1 \mathrm{~m}, 0 \mathrm{~m}, 0.005 \mathrm{~m})$. Since this case is more complex than the precedent straight pipe, the coupling between the $\mathrm{BEM}$ and the analytical 2D computations of section 2 is used in this case.

The results at $(1 \mathrm{~m}, 0 \mathrm{~m}, 0.06 \mathrm{~m})$ are shown in Fig.7a where we can see two obvious variations of SPL around the resonant frequencies compared to the solid line. The first variations are reductions around $1220 \mathrm{~Hz}$, and the second variations are reductions and 


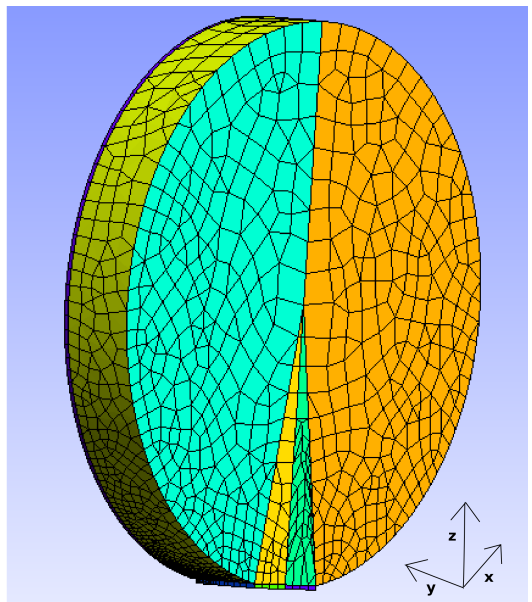

(a)

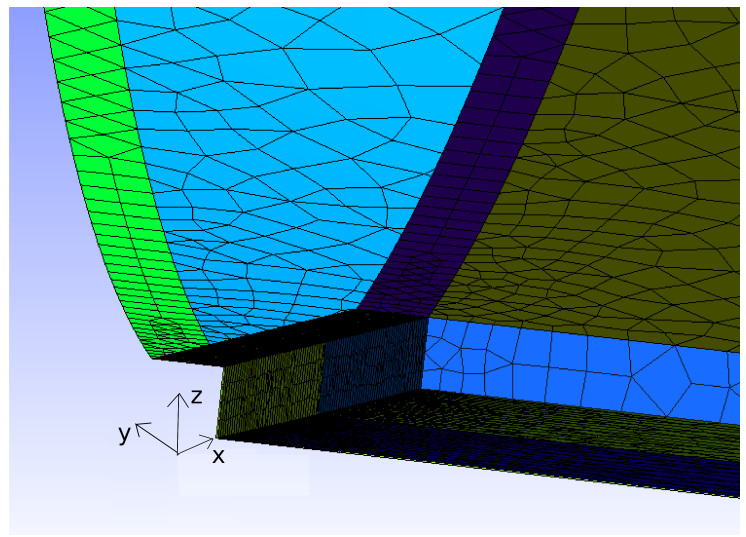

(b)

Figure 5: (a) Meshes of half a cylinder with half of a straight pipe at the center of the contact zone; (b) Details of meshes of the contact zone.

amplifications around $1850 \mathrm{~Hz}$. From table 1 we can see the influences of viscous and thermal conduction losses on the SPL. At the frequencies of three SPL extrema, the more losses we take into account, the smaller the SPL differences between the case without network and the case with one network are.

Table 1 Differences of SPL in Fig.7a between the case without network and the case with a network around the resonant frequencies

\begin{tabular}{cccc}
\hline Frequencies & No losses & Viscous losses & VT losses \\
\hline $1220 \mathrm{~Hz}$ & $3.30 \mathrm{~dB}$ & $2.98 \mathrm{~dB}$ & $2.85 \mathrm{~dB}$ \\
$1800 \mathrm{~Hz}$ & $6.89 \mathrm{~dB}$ & $5.41 \mathrm{~dB}$ & $4.88 \mathrm{~dB}$ \\
$1880 \mathrm{~Hz}$ & $3.13 \mathrm{~dB}$ & $2.65 \mathrm{~dB}$ & $2.44 \mathrm{~dB}$ \\
\hline \multicolumn{2}{c}{ Note: Viscous and thermal conduction losses (VT losses) }
\end{tabular}




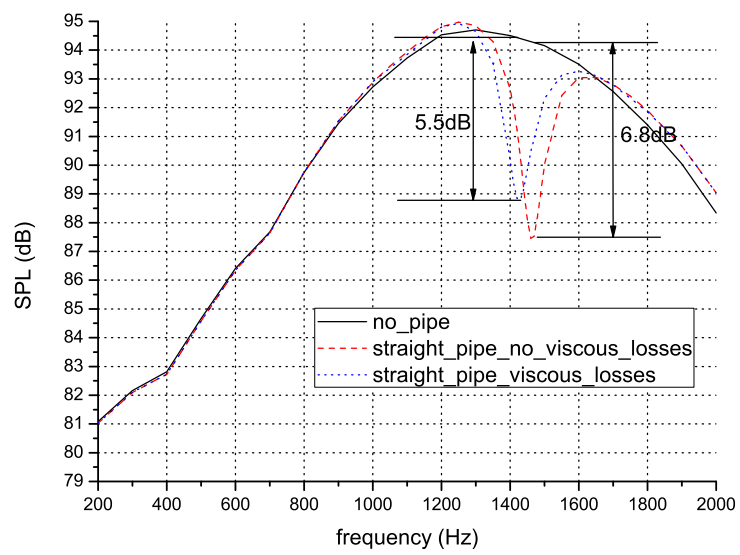

Figure 6: Predicted results for a straight pipe between a cylinder and a plane surface

The influence of the network on the acoustic fields mainly results from the pipe resonances. The viscous and thermal conductivity losses can give small corrections. If no viscous and thermal conductivity losses are taken into account, the multi-domain coupling methods can still be applied to the estimation of the tendencies of the acoustic pressure and the resonant frequencies.

\subsection{Experimental validation}

The flange-network interaction model has been established. In this section, experiments will be carried out to validate the numerical simulations of this model. The radius and width of the cylinder in Fig.9b are $0.27 \mathrm{~m}$ and $0.15 \mathrm{~m}$. Its location can be seen in Fig. 8 where the sketch of the experimental setup is shown. The locations of the source and receiver are exchanged in Fig.9a thanks to the reciprocity principle. The experiments are performed in a large hall to avoid a too large influence of the reflections from the wall. The plywood on the floor in Fig.9a is used to simulate a rigid plane surface. Sweep signals from $1 \mathrm{~Hz}$ to $2000 \mathrm{~Hz}$ are generated by a generator. The center of the speaker is at $(1 \mathrm{~m}, 0 \mathrm{~m}, 0.06 \mathrm{~m})$. The microphone is at $(0.1 \mathrm{~m}, 0 \mathrm{~m}, 0.005 \mathrm{~m})$.

First we present the acoustic source used in our experiments. Then the acoustic fields of a cylinder on a plane surface are computed and measured. In this comparison, there is no pipe between the cylinder and the plane surface. Next a straight pipe between the cylinder and the plane surface are measured. Last a network between the cylinder and the plane surface is measured.

\subsubsection{Point source}

In our simulations, a dimensionless point source is used, and it has been assumed omnidirectional. Typical sources used in previous researches are audio speakers $\mathrm{Yu}$ (2009), audio speakers coupled with circular pipe Jin et al (2001) and electrostatic spark sources Jolibois (2013). In our study the predicted results will not be compared directly with the measured results. Instead, the general tendencies and frequency-dependant variations of the sound pressure will be compared between the predicted and measured results. Such comparisons are very common and widely used in previous studies such as in Cummings (1992) and Yu 


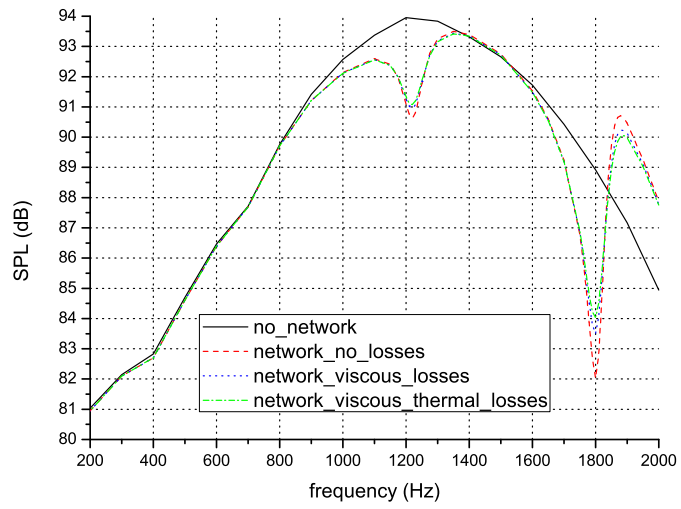

(a)

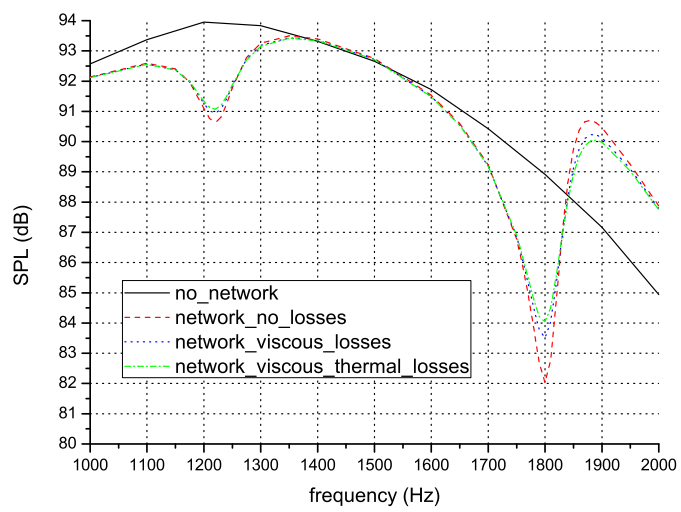

(b)

Figure 7: Predicted results for a network between a cylinder and a plane surface: (a) 200 $2000 \mathrm{~Hz}$; (b) Around the resonant frequencies

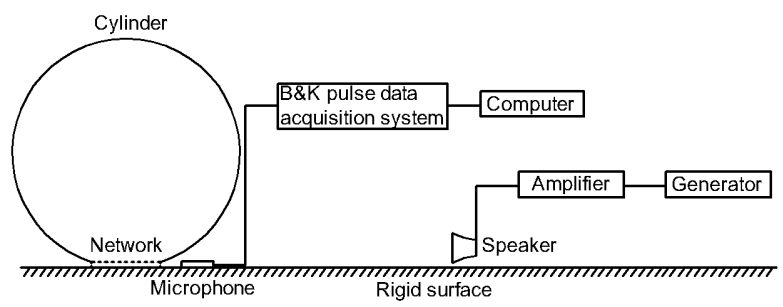

Figure 8: Sketch of the experimental setup

(2009). So, it is not necessary to use a omni-directional source in our experiments. A real loudspeaker, whose signal is digitally generated, is used as source in the experiments. Its dimensions are $0.12 \mathrm{~m} \times 0.12 \mathrm{~m} \times 0.12 \mathrm{~m}$. 


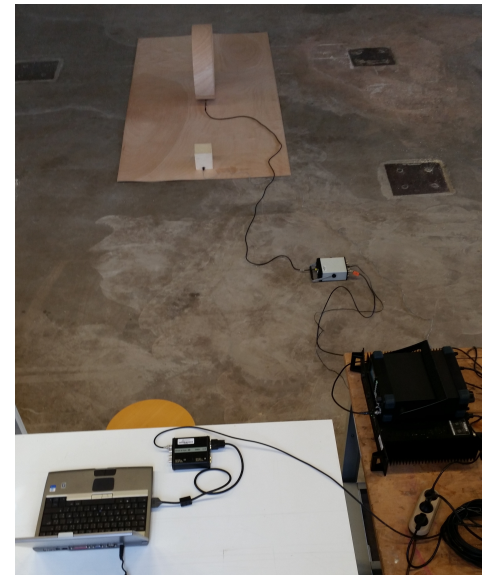

(a)

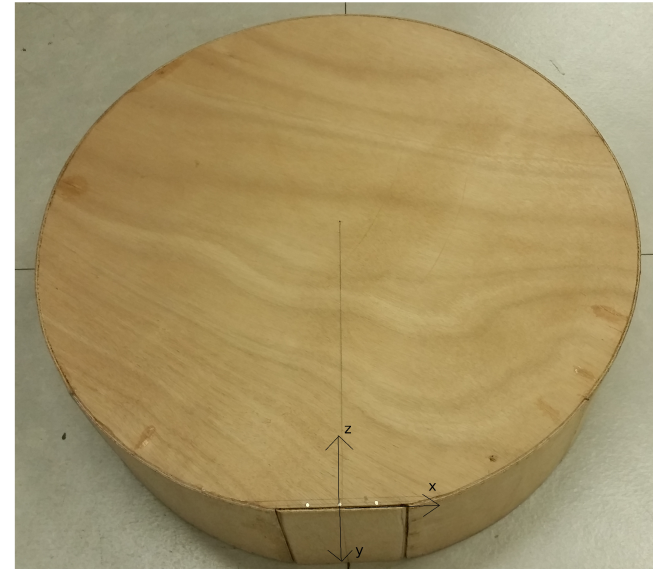

(b)

Figure 9: (a) The experimental set-up; (b) flange used in the calculations and experiments

\subsubsection{Plane surface with or without a cylinder}

Since the measurements are performed in a large hall with walls not fully anechoic, it is essential to know if the influence of reflections from walls and roof can be ignored or not. In this section, two measurements are done for this purpose. In the first case, a cylinder is located on a plane surface, but there is no pipe between the cylinder and the plane surface. In the second case, we move the cylinder away from the plane surface.

The acoustic field of the first case can be calculated by BEM. In the second case the plane surface is rigid, so it can be considered as a symmetric plane. An image source is created in Fig.10b. Thus, the second case can be solved by equations (26) and (27). The predicted results can be seen in Fig.11a. The measured results of SPL are shown in Fig.11b.

$$
p=\frac{e^{i k d_{1}}}{4 \pi d_{1}}+\frac{e^{i k d_{2}}}{4 \pi d_{2}}
$$

where $d_{1}$ and $d_{2}$ are the distances from the source and the image source to the receiver in Fig. $10 b$.

$$
S P L=10 \log _{10} \frac{|p|^{2}}{4 \times 10^{-10}}
$$

When we compare the predicted results in Fig.11a and the measured results in Fig.11b, similar tendencies of SPL can be found. The maximum difference of predicted SPL between the case with the cylinder and the case without the cylinder is about $15 \mathrm{~dB}$ around $1250 \mathrm{~Hz}$ in Fig.11a. The maximum difference between these two cases of the measured SPL is about $14.5 \mathrm{~dB}$ around $1300 \mathrm{~Hz}$ in Fig.11b. The predicted results agree well with the measured results. We can conclude that the reflections from the walls and the roof can be ignored in our experiments. 


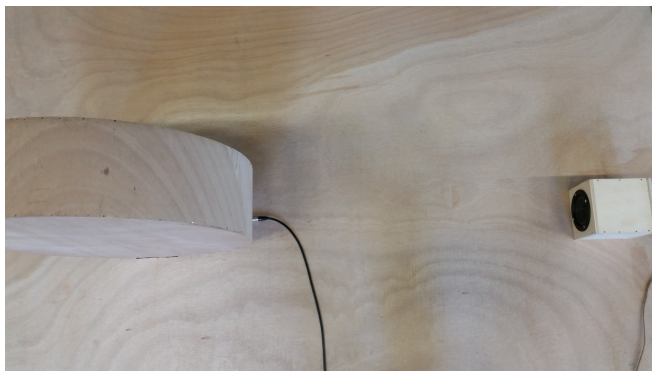

(a)

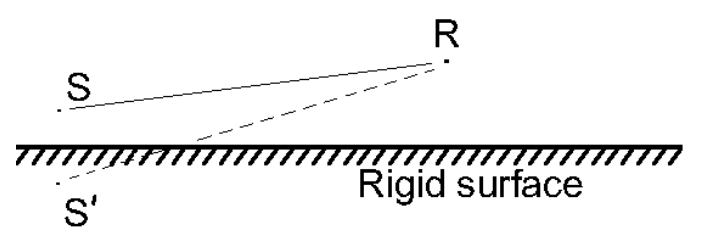

(b)

Figure 10: (a) A cylinder on a plane surface (no pipe between the cylinder and the plane surface); (b) A rigid plane surface between a source and an image source.

\subsubsection{Straight pipe}

The example calculated in section 3.1.1 is measured in this section, which can be seen in Fig.12. We measured the case with a straight pipe and the case without pipe. In the case without pipe, we close the pipe ends with woods.

The results are shown in Fig.13, where we can see a reduction of SPL of 3.5dB around the resonant frequency $1460 \mathrm{~Hz}$. The reduction of numerical results with viscous losses in Fig. 6 is $5.5 \mathrm{~dB}$ at the resonant frequency $1430 \mathrm{~Hz}$. The difference of reduction at the resonant frequency between measurement and simulation is $2 \mathrm{~dB}$. In this example the tendencies of numerical SPL are similar to the measurements, and the resonant frequency is estimated correctly.

\subsubsection{Network}

The network calculated in section 3.1.2 is measured in this part to validate the multi-domain coupling methods proposed in this work. When we measure the case without network, we close the pipe ends in Fig.14 with woods.

Table 2 Measured results

\begin{tabular}{|c|c|}
\hline Measured f & Measured differences \\
\hline $1200 \mathrm{~Hz}$ & $4 \mathrm{~dB}$ \\
$1800 \mathrm{~Hz}$ & $3.5 \mathrm{~dB}$ \\
$1900 \mathrm{~Hz}$ & $3.2 \mathrm{~dB}$ \\
\hline
\end{tabular}

The experiment is shown in Fig.14. The results can be seen in Fig.15. There are three SPL extrema around the two resonant frequencies. Around the first resonant frequency, we can see a reduction. Around the second resonant frequency, there are a reduction and an 


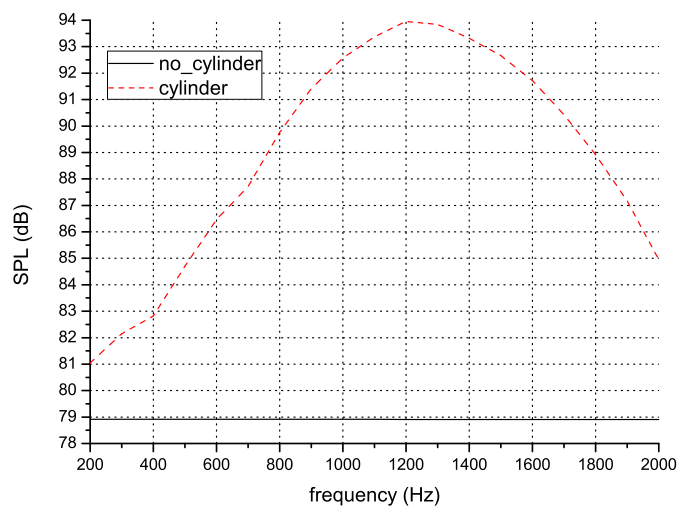

(a)

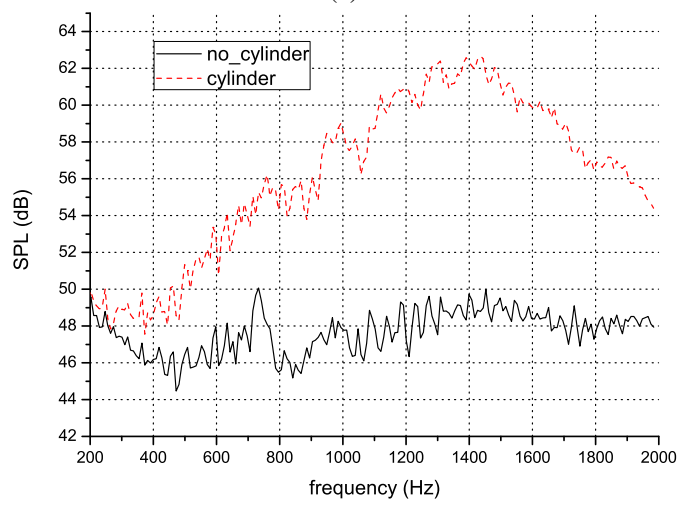

(b)

Figure 11: (a) Predicted results; (b) Measured results.

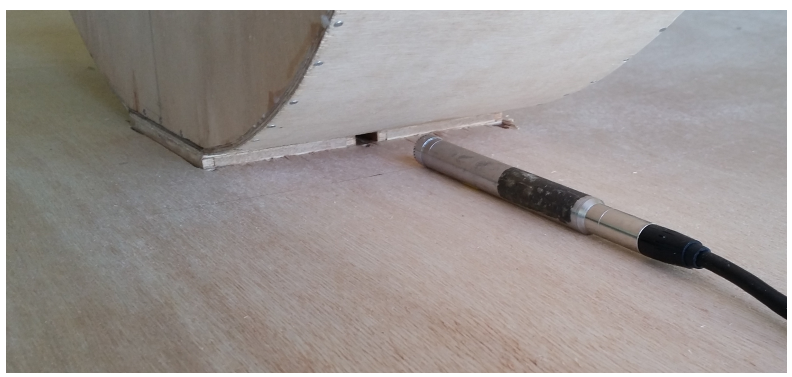

Figure 12: A straight pipe between a cylinder and a plane surface

amplification. The measured frequencies of these three extrema and the differences between the case with pipes and the case without pipe are shown in table 2 . This is to be compared with the predicted results of table 1 .

At the second extremum at the frequency $1800 \mathrm{~Hz}$, the SPL reduction of experimental results between the solid line and the dash line is about $3.5 \mathrm{~dB}$. The SPL reduction $4.88 \mathrm{~dB}$ of numerical results with viscous and thermal conduction losses is closer to the measurements. 


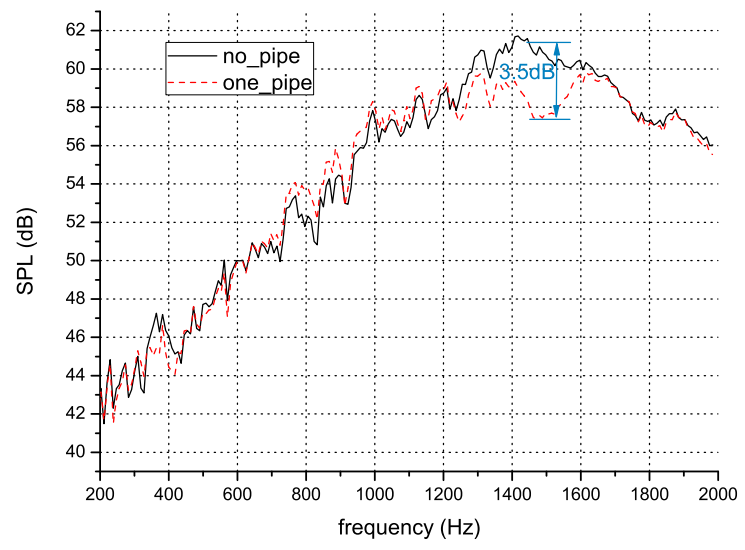

Figure 13: Measured results of flange with a straight pipe

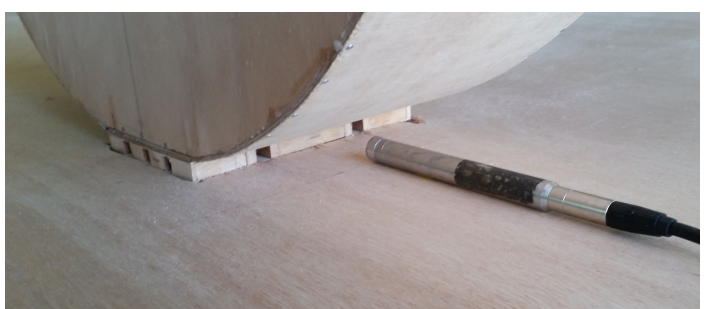

Figure 14: A network between a cylinder and a plane surface

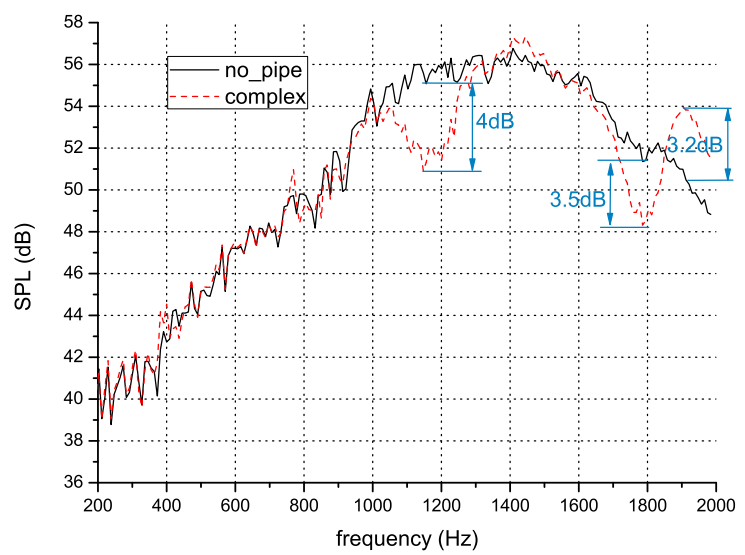

Figure 15: Measured results of flange with a network

So for a thin pipe in this work, the viscous and thermal conduction losses should be taken into account. Otherwise, the difference of reductions between experimental and numerical results for the second extremum is too large. For the two other resonance frequencies the differences are smaller and of the order of the measurement error. Compared with simulations, the frequencies of these three extrema, which are about $1200 \mathrm{~Hz}, 1800 \mathrm{~Hz}$ and 
$1900 \mathrm{~Hz}$, are estimated correctly, and the variations of these three extrema are also very close. The errors are $1.15 \mathrm{~dB}, 1.38 \mathrm{~dB}$ and $0.76 \mathrm{~dB}$. Besides, we can see similar tendencies of SPL between experiments and simulations.

\section{Application to horn effect of tire/road noise}

In order to know whether the tire treads in the contact zone have influences on the acoustic radiation of the tire and road system, measurements of the acoustic fields for a real Michelin $165 / 65$ R 13 tire are performed. Then the calculations of the sound pressures are done by the methods proposed in the previous sections. The tire treads in Fig.16a are very complex. There

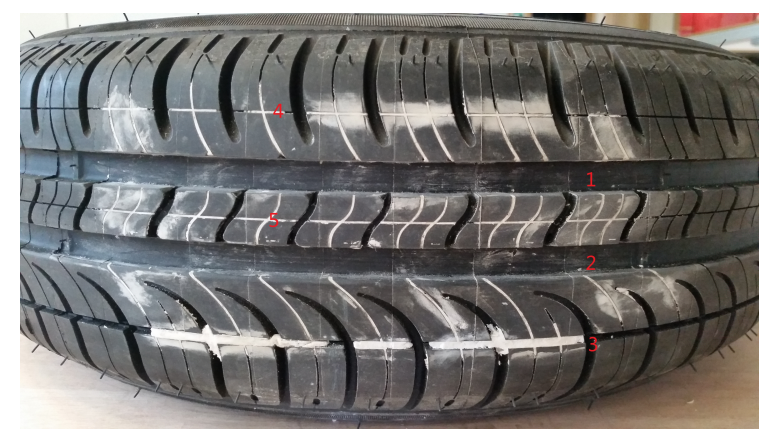

(a)

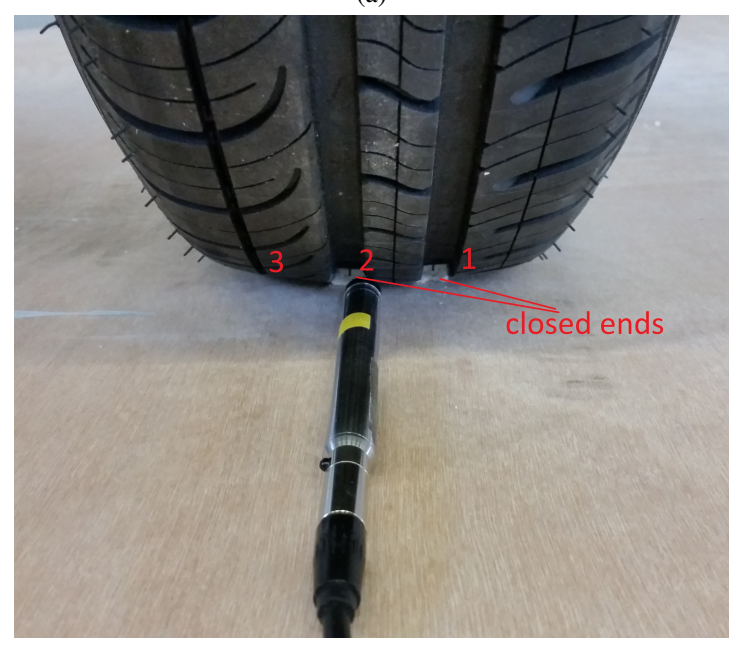

(b)

Figure 16: (a) Tire with an open network; (b) Tire with a closed network.

are three longitudinal pipes (pipe 1,2 and 3) with large cross-sections, two longitudinal pipes (pipe 4 and 5 ) with very small cross-sections and many transverse pipes with different cross-sections. For the sake of simplicity, we only investigate the network in the middle of the contact zone. The network consists of the longitudinal pipes 1 and 2 and the large transverse pipes between them in Fig.16a. But there are also many small pipes connecting 
to the network to be studied, so we fill them with silicone. We also fill the longitudinal pipe 3 to make sure that there is no other longitudinal pipe except the pipe 1 and 2 in the network to be investigated.

Two tests are performed. In the first test, the network is open. In the second test, we fill the network with silicone in the contact zone (see Fig.16b). We can see that the ends of the pipes 1 and 2 are closed in Fig.16b. These two tests are compared to study the influence of the treads on the acoustic radiation. We use five concrete cylinders (Fig.17a) as a load of approximately $74 \mathrm{~kg}$ to get the flat contact zone (Fig.17b). The purpose is not to simulate the real contact zone of a tire of a wheel mounted on a car but to get a simple experiment with this sort of tire. In order to obtain the dimensions of the network in the contact zone, first we put the tire on the flour for several minutes. Next we move the tire on a clean surface. Then we lay the tire flat on the surface. In Fig.17c we can see the elliptical contact zone which is given by the lumps of flour on the tire or on the road. When we compare the tire with and without the lumps of flour in Fig.18, we can see clearly the shape of the contact zone. The length of the contact zone is $0.161 \mathrm{~m}$. The experimental setup is the same as the wooden cylinder case, which is shown in Fig.8. The source is at $(1 \mathrm{~m}, 0 \mathrm{~m}, 0.06 \mathrm{~m})$, and the receiver is at $(0.15 \mathrm{~m},-0.005 \mathrm{~m}, 0.005 \mathrm{~m})$.

In order to predict the influence of the tire treads on the acoustic fields of our tire and road systems, the network is modeled as the one in Fig.19a. The BEM meshes of the tire can be seen in Fig.19b. We ignore the transverse grooves on the tire surface except the ones in the network to be studied. We only take into account the two large longitudinal pipes 1 and 2. The other longitudinal pipes are not modeled and the lateral surface in the model is a flat surface. The model is mainly used to compare the cases with and without the network and estimate the influence of the network on the acoustic fields so all the geometric details are not taken into account. In the calculations of these two cases, the network to be investigated will be changed from open ends to closed ends, but the other parts keep the same. The tire width and radius are $0.165 \mathrm{~m}$ and $0.27 \mathrm{~m}$, respectively. The contact zone is $0.161 \mathrm{~m} \times 0.165 \mathrm{~m}$. This is a little larger than the real contact zone of a rolling tire but the purpose here is to estimate the physical phenomenon and not to make precise predictions for a rolling tire. Two calculations are done. In the first calculation, the ends of the network are open. We use the multi-domain methods given before to solve the problem. In the second calculation, the ends of the network are closed. We use the BEM to do the calculations.

Sound pressures with and without network are presented in Fig.20a for the simulation and the measurement at receiver $(0.15 \mathrm{~m},-0.005 \mathrm{~m}, 0.005 \mathrm{~m})$. Note that the scales are different between the computed and measured sound pressures but the purpose is only to compared the difference between the cases with and without network and not to adjust the global levels. From the measured sound pressures in Fig.20a we can see only one resonant frequency around $1734 \mathrm{~Hz}$. There is no variation of SPL around $868 \mathrm{~Hz}$ which should be another resonance as seen in Fig.20b. Maybe because the tire is not pressed firmly in the whole contact zone. When we did the tests of wooden networks, we found that if the woods are not glued firmly no resonant frequency can be found. The general tendencies of the predicted SPL are not quite similar to the measured results. The reason is that the BEM model in Fig.19b is not exactly the same as the real tire, for example the lateral surface. But the influence of the network on the acoustic fields around $1734 \mathrm{~Hz}$ are estimated approximately. Around the resonant frequency $1734 \mathrm{~Hz}$ of the network, we can see the variations of SPL in Fig.20b. In Fig.20a, we can see the similar variations around $1734 \mathrm{~Hz}$. But errors of reductions and amplifications between the measured and predicted results can be seen. These errors are due to the differences between the real tire and the 


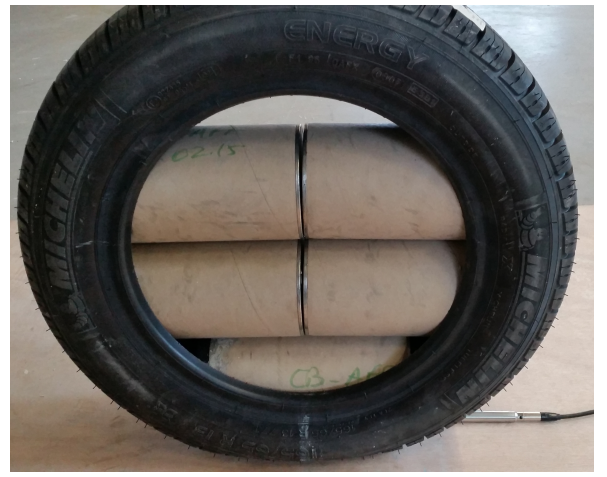

(a)

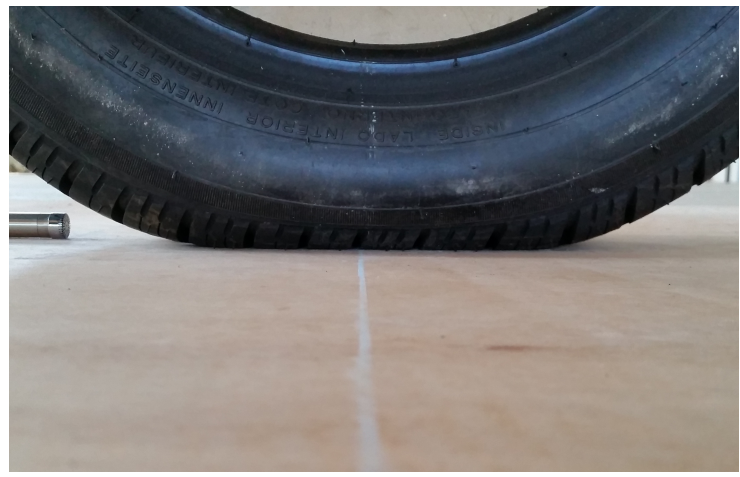

(b)

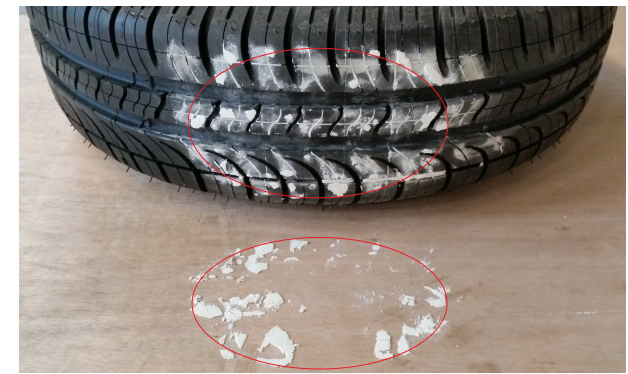

(c)

Figure 17: (a) The load of five concrete cylinders; (b) The contact zone between the tire and the road; (c) The contact area given by the flour.

simplified model that we use. Although above $1000 \mathrm{~Hz}$ the agreement between the measured and predicted results in our case of real tire is not as perfect as the agreement in the case of the wooden cylinder and the wooden networks, we can still use our proposed model and methods to get an estimate of the influence of the tire treads on the acoustic fields and on the ways it can change the horn effect. 


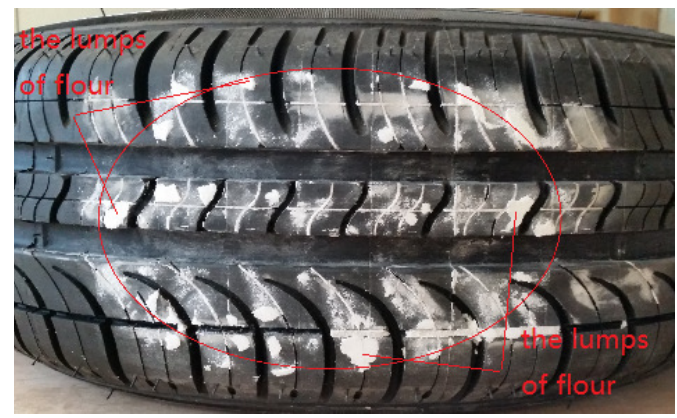

Figure 18: The tire with the lumps of flour

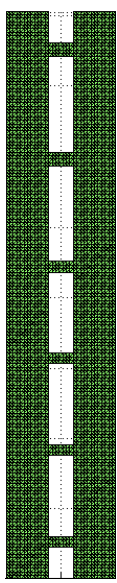

(a)

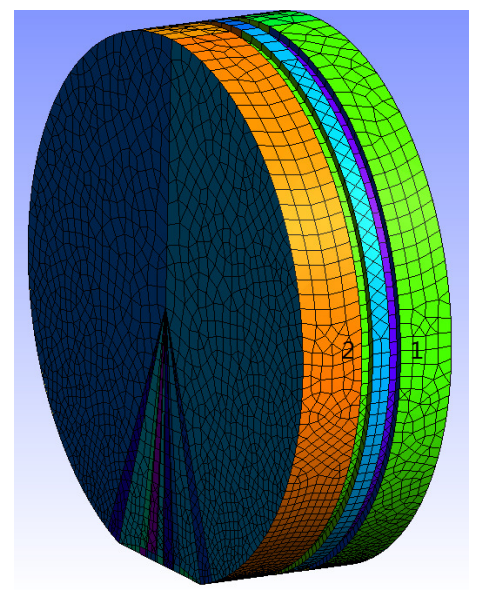

(b)

Figure 19: (a) The simplified network; (b) The meshes of the BEM model of the tire used in the multi-domain coupling methods.

\section{Conclusions}

The tire/road system and the networks in the contact zone can be considered as flanged networks. Multi-domain coupling methods are developed for the computation. We use wooden pipes to compare the predicted results and the measured results. The availability and effectiveness of the methods are shown by a reasonable agreement between the computations and the measurements. For a thin pipe in this work, the viscous and thermal conduction losses should be taken into account. Then a real tire is measured and calculated. Since it is difficult to estimate the dimension of the network in the contact zone, the agreement between the predicted and measured results is not as perfect as the agreement in the wooden cases. However, we can see the influence of the tire treads on the acoustic fields from the experimental results, and our proposed network can be used for the estimation of the tread of the tire on the horn effect. 


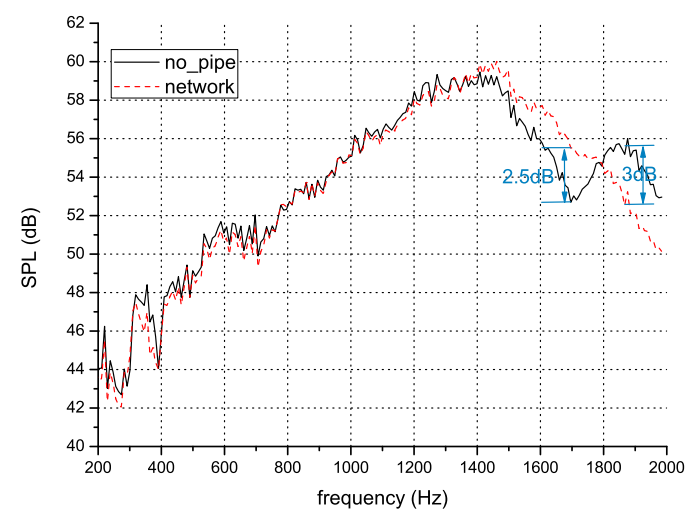

(a)

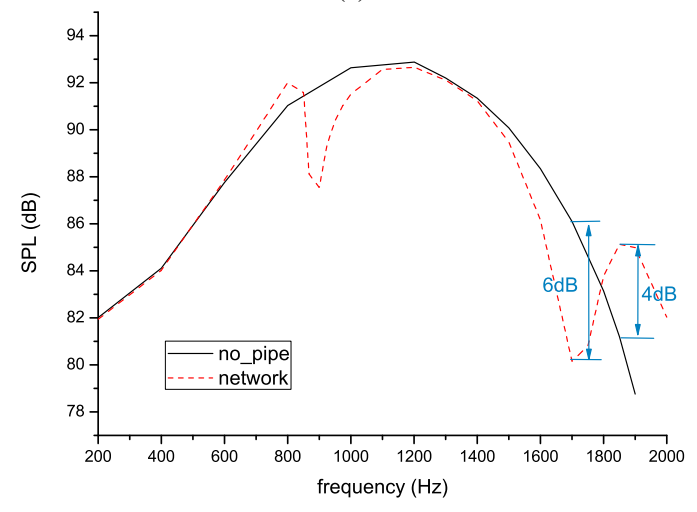

(b)

Figure 20: (a) Measured results of the network between a tire and a plane surface; (b) Predicted results of the network between a tire and a plane surface.

\section{References}

Anfosso-Ledee, F., Klein, P., Fadavi, A. and Duhamel, D. (2000) 'Tire/road noise: comparison of $2 \mathrm{~d}$ and $3 \mathrm{~d}$ models for horn effect', in Internoise 2000. Proceedings of the 29th international congress on noise control engineering, 27-31 August 2000. Nice, France.

Anfosso-Ledee, F., Klein, P., Fadavi, A. and Duhamel, D. (2000), 'Tyre/road noise horn effect: $2 \mathrm{~d}$ modelling by analytical and numerical models' in 7th International conference on sound and vibration. 4-7 July 2000, Vol. 5, pp.2789-2796. Garmisch-Partenkirchen, Germany.

Berglund, B., Lindvall, T. and Schwela, D.H. (1999) Guidelines for community noise. in Guidelines for community noise, OMS.

Bernhard, R., Wayson, R.L., Haddock, J., Neithalath N., El-Aassar A., Olek J., Pellinen T. and Weiss W.J. (2005). An introduction to tire/pavement noise of asphalt pavement, Institute of Safe, Quiet and Durable Highways, Purdue University. 
Bonnet, M. (1999) Boundary integral equation methods for solids and fluids, Wiley, Chichester, England.

Bravo, T. (2017) 'An analytical study on the amplification of the tyre rolling noise due to the horn effect', Applied Acoustics, Vol. 123, pp.85-92.

Bravo, T. and de la Colina, C. (2015) 'An analytical study for the prediction of the horn effect above a ground surface', in The 22nd International congress on sound and vibration, 12-16 July, 2015. Florence, Italy.

Brebbia, C.A. and Walker, S. (1980) Boundary Element Techniques in Engineering, Newnes-Butterworths.

Cheng, C.R. (1988) Boundary Element Analysis of Single and Multidomain Problems in Acoustics. PhD thesis, University of Kentucky.

Cheng, C.Y.R., Seybert, A.F. and Wu, T.W. (1991). 'A multidomain boundary element solution for silencer and muffler performance prediction', Journal of Sound and Vibration, Vol. 151 No. 1, pp.119-129.

Chen, G. and Zhou, J. (1992) Boundary element methods, Computational mathematics and applications, Academic press, London, England.

Ciskowski, R.D. and Brebbia, C.A. (1991) Boundary element methods in acoustics. Computational mechanics publications, Elsevier, Southampton, England.

Craggs, A. and Stredulinsky, D.C. (1990) 'Analysis of acoustic wave transmission in a piping network', The Journal of the Acoustical Society of America, Vol. 88, pp.542-547.

Cummings, A. (1992) 'The effects of a resonator array on the sound field in a cavity' Journal of Sound and Vibration, Vol. 154 No. 1, pp.25-44.

Duhamel, D. (1995) 'Static regularization of hypersingular integral equations in acoustics', in 3ème conférence internationale Mathematical and numerical aspects of wave propagation, pp.198-207, 24-28 April 1995. Mandelieu-La-Napoule, France.

Duhamel, D. (1994) L'acoustique des problèmes couples fluide-structure-Application au contrôle actif du son, $\mathrm{PhD}$ thesis, Ecole des Ponts ParisTech.

European commission (1996) Future noise policy, European commission green paper, com (96) 540 final, 4 november 1996.

Gagen, M.J. (1999) 'Novel acoustic sources from squeezed cavities in car tires', The Journal of the Acoustical Society of America, Vol. 106 No. 2, pp.794-801.

Graf, R.A.G., Kuo, C.Y., Dowling, A.P. and Graham, W.R. (2002) 'On the horn effect of a tyre/road interface, part i: Experiment and computation', Journal of Sound and Vibration, Vol. 256 No. 3, pp.417-431.

Jin, B.J., Kim, H.S., Kang, H.J. and Kim, J.S. (2001) 'Sound diffraction by a partially inclined noise barrier', Applied acoustics, Vol. 62 No. 9, pp.1107-1121.

Jolibois, A. (2013) A study on the acoustic performance of tramway low-height noise barriers: gradient-based numerical optimization and experimental approaches, $\mathrm{PhD}$ thesis, Université Paris Est. 
Kinsler, L.E., Frey, A.R., Coppens, A.B. and Sanders, J.V. (1999) Fundamentals of Acoustics, 4th Edition, Wiley-VCH, December 1999.

Kuo, C.Y., Graf, R.A.G., Dowling, A.P. and Graham, W.R. (2002) 'On the horn effect of a tyre/road interface, part ii: Asymptotic theories', Journal of sound and vibration, Vol. 256 No. 3, pp.433-445.

Lambert, J. and Vallet, M. (1994) Study related the preparation of a communication on a future EC noise policy, report no. len 9420, december 1994, INRETS, Bron Cedex, France.

Lou, G., Wu, T.W. and Cheng, C.Y.R. (2003) 'Boundary element analysis of packed silencers with a substructuring technique', Engineering Analysis with Boundary Elements, Vol. 27 No. 7, pp.643-653.

Miles, J.W. (1946) 'The analysis of plane discontinuities in cylindrical tubes. part i.', The Journal of the Acoustical Society of America, Vol. 17 No. 3, pp.259-271.

Miles, J.W. (1946) 'The analysis of plane discontinuities in cylindrical tubes. part ii.', The Journal of the Acoustical Society of America, Vol. 17 No. 3, pp.272-284.

Miles, J.W. (1947) 'The diffraction of sound due to right-angled joints in rectangular tubes', The Journal of the Acoustical Society of America, Vol. 19 No. 4, pp.572-579.

Munjal, M.L. (1987) Acoustics of ducts and mufflers with application to exhaust and ventilation system design, Wiley New York (NY).

Park, Y.B., Ju, H.D. and Lee, S.B. (2009) 'Transmission loss estimation of three-dimensional silencers by system graph approach using multi-domain boundary element method', Journal of Sound and Vibration, Vol. 328 No. 4, pp.575-585.

Qatu, M.S., Abdelhamid, M.K., Pang, J. and Sheng, G. (2009) 'Overview of vehicle noise and vibration', International Journal of Vehicle Noise and Vibration, Vol. 5, No. 1-2, pp.1-35

Qatu, M.S. (2012) 'Recent research on vehicle noise and vibration', International Journal of Vehicle Noise and Vibration, Vol. 8, No. 4, pp.289-301

Utsuno, H., Wu, T.W., Seybert, A.F. and Tanaka, T. (1990) 'Prediction of sound fields in cavities with sound absorbing materials', AIAA Journal, Vol. 28, pp.1870-1876.

Wang, C.N., Tse, C.C. and Chen Y.N. (1993) 'A boundary element analysis of a concentrictube resonator', Engineering analysis with boundary elements, Vol. 12 No. 1, pp.21-27.

Wu, T.W. (2008) 'Multi-domain boundary element method in acoustics', in Computational Acoustics of Noise Propagation in Fluids-Finite and Boundary Element Methods, pp.367386, Springer.

Yu, G. (2009) Acoustic resonators for noise control in enclosures: modelling, design and optimization, PhD thesis, The Hong Kong Polytechnic University.

Zhang, Y., Bi, C.X., Zhang, Y.B. and Zhang, X.Z. (2017) 'Horn effect prediction based on the time domain boundary element method', Engineering Analysis with Boundary Elements, Vol. 82, pp.79-84. 